\title{
Higher spin Dirac operators between spaces of simplicial monogenics in two vector variables
}

\author{
F. Brackx*, D. Eelbode ${ }^{\ddagger}$, L. Van de Voorde* \\ * Clifford Research Group, Department of Mathematical Analysis, Faculty of Engineering, Ghent \\ University, Belgium \\ $¥$ Department of Mathematics and Computer Science, University of Antwerp, Campus \\ Middelheim, G-Building, Middelheimlaan 1, 2020 Antwerpen, Belgium
}

\begin{abstract}
The higher spin Dirac operator $\mathcal{Q}_{k, l}$ acting on functions taking values in an irreducible representation space for $\mathfrak{s o}(m)$ with highest weight $\left(k+\frac{1}{2}, l+\frac{1}{2}, \frac{1}{2}, \ldots, \frac{1}{2}\right)$, with $k, l \in \mathbb{N}$ and $k \geq l$, is constructed. The structure of the kernel space containing homogeneous polynomial solutions is then also studied.

2000 Mathematics Subject Classification: 15A66, 30G35, 22E46
\end{abstract}

\section{Introduction}

Consider an oriented spin manifold, i.e. a Riemannian manifold with a spin structure which allows the construction of vector bundles whose underlying symmetry group is $\operatorname{Spin}(m)$ rather than $S O(m)$, see e.g. [17]. On such a Riemannian spin manifold there is a whole system of conformally invariant, elliptic, first-order differential operators acting on sections of an appropriate spin bundle, whose existence and construction can be established through geometrical and representation theoretical methods, see e.g. [5, 13, 21, 22]. In Clifford analysis these operators are studied from a function theoretical point of view, mainly focusing on their rotational invariance with respect to the spin group $\operatorname{Spin}(m)$, or its Lie algebra $\mathfrak{s o}(m)$, and considering functions on $\mathbb{R}^{m}$ instead of sections. The simplest example is the Dirac operator acting on spinor-valued functions; we refer to the standard references $[1,11,15]$. Next in line are the Rarita-Schwinger operator, acting on functions with values in the irreducible $\mathfrak{s o}(m)$-representation with highest weight $\left(\frac{3}{2}, \frac{1}{2}, \ldots, \frac{1}{2}\right)$, and its generalizations to the case of functions taking values in irreducible representation spaces with highest weight $\left(k+\frac{1}{2}, \frac{1}{2}, \ldots, \frac{1}{2}\right)$, see e.g. [7, 8]. Also higher spin Dirac operators acting on spinor-valued forms have been studied in detail, see e.g. [6, 20].

Our aim is to combine techniques from Clifford analysis and from representation theory, in order to investigate, from the function theoretical point of view, general higher spin Dirac operators acting between functions taking values in an arbitrary finite-dimensional half-integer highest-weight representation. As the case of the Rarita-Schwinger operator (and its generalizations) does not yet contain the seed from which the most general case can 
be derived, we study, in this paper, the particular case of the operator acting on functions taking values in the irreducible representation with highest weight $\left(k+\frac{1}{2}, l+\frac{1}{2}, \frac{1}{2}, \ldots, \frac{1}{2}\right)$, with $k, l \in \mathbb{N}$ and $k \geq l$. This is done using the elegant framework of Clifford analysis in several vector variables.

\section{Clifford analysis and definitions}

Let $\left(\underline{e}_{1}, \ldots, \underline{e}_{m}\right)$ be an orthonormal basis for the Euclidean space $\mathbb{R}^{m}$. We denote by $\mathbb{C}_{m}$ the complex universal Clifford algebra, generated by these basis elements, its multiplication being governed by the relations $\underline{e}_{i} \underline{e}_{j}+\underline{e}_{j} \underline{e}_{i}=-2 \delta_{i j}, i, j=1, \ldots, m$. The space $\mathbb{R}^{m}$ is embedded in $\mathbb{C}_{m}$ by identifying $\left(x_{1}, \ldots, x_{m}\right)$ with the real Clifford vector $\underline{x}=\sum_{j=1}^{m} \underline{e}_{j} x_{j}$. The multiplication of two vectors $\underline{x}$ and $\underline{y}$ is given by $\underline{x} \underline{y}=-\langle\underline{x}, \underline{y}\rangle+\underline{x} \wedge \underline{y}$ with

$$
\langle\underline{x}, \underline{y}\rangle=\sum_{j=1}^{m} x_{j} y_{j} \quad \text { and } \quad \underline{x} \wedge \underline{y}=\sum_{1 \leq i<j \leq m} \underline{e}_{i} \underline{e}_{j}\left(x_{i} y_{j}-x_{j} y_{i}\right)
$$

the scalar-valued Euclidean inner product and the bivector-valued wedge product respectively. The wedge product of a finite number of vectors in $\mathbb{R}^{m}$ may also be defined using the Clifford product:

Definition 1. The wedge product of $N$ Clifford vectors $\underline{x}_{1}, \ldots, \underline{x}_{N}$ is defined as

$$
\underline{x}_{1} \wedge \ldots \wedge \underline{x}_{N}:=\frac{1}{N !} \sum_{\sigma \in S_{N}} \operatorname{sgn}(\sigma) \underline{x}_{\sigma(1)} \ldots \underline{x}_{\sigma(N)},
$$

where $S_{N}$ denotes the symmetric group in $N$ elements.

For convenience, we will work in odd dimension $m=2 n+1$. In this case there is a unique spinor space $\mathbb{S}$, as opposed to the even-dimensional case $m=2 n$ where there are two spinor representations (often referred to as even and odd spinors). However, these cases do not differ from each other conceptually: in case of even dimension $m=2 n$, it suffices to take into account that the vector-valued (higher spin) Dirac operator will change the parity of the underlying values. The spinor space $\mathbb{S}$ should be thought of as a minimal left ideal in $\mathbb{C}_{m}$, which can be defined in terms of a primitive idempotent; it is characterized by the highest weight $\left(\frac{1}{2}, \ldots, \frac{1}{2}\right)$ under the standard multiplicative action of the spin group

$$
\operatorname{Spin}(m)=\left\{\prod_{j=1}^{2 k} s_{j}: k \in \mathbb{N}, s_{j} \in S^{m-1}\right\},
$$

with $S^{m-1}$ the unit sphere in $\mathbb{R}^{m}$. In case one prefers working with its Lie algebra $\mathfrak{s o}(m)$, which can be identified with the subspace of bivectors in the algebra $\mathbb{C}_{m}$, the derived action should be used.

The Dirac operator is denoted $\underline{\partial}_{x}=\sum_{j=1}^{m} \underline{e}_{j} \partial_{x_{j}}$. It is an elliptic $\operatorname{Spin}(m)$-invariant firstorder differential operator acting on spinor-valued functions $f(\underline{x})$ on $\mathbb{R}^{m}$. It factorizes the 
Laplace operator: $\Delta_{x}=-\underline{\partial}_{x}^{2}$ on $\mathbb{R}^{m}$. An $\mathbb{S}$-valued function $f$ is monogenic in an open region $\Omega \subset \mathbb{R}^{m}$ if and only if it satisfies $\underline{\partial}_{x} f=0$ in $\Omega$. For a detailed account of the theory of monogenic functions, so called Euclidean Clifford analysis, we refer the reader to e.g. $[1,11,15]$. We also mention the Euler operator $\mathbb{E}_{x}=\sum_{i} x_{i} \partial_{x_{i}}$, measuring the degree of homogeneity in the variable $\underline{x}$.

Irreducible (finite-dimensional) modules for the spin group can be described in terms of spaces of traceless tensors satisfying certain symmetry conditions expressed in terms of Young diagrams, see e.g. $[14,16]$, but they can also be realized in terms of vector spaces of polynomials, see e.g. [10, 15]. We mention the following well-known examples from harmonic and Clifford analysis: the vector space $\mathcal{H}_{k}$ of $\mathbb{C}$-valued harmonic homogeneous polynomials of degree $k \in \mathbb{N}$ corresponds to the irreducible $\operatorname{Spin}(m)$-module with highest weight $(k, 0, \ldots, 0)$, and the vector space $\mathcal{M}_{k}$ of spinor-valued monogenic homogeneous polynomials of degree $k$ forms an irreducible representation of $\operatorname{Spin}(m)$ with highest weight $\left(k+\frac{1}{2}, \frac{1}{2}, \ldots, \frac{1}{2}\right)$.

In what follows, $N \in \mathbb{N}$ and $\underline{\partial}_{i}$ is short for the Dirac operator $\underline{\partial}_{u_{i}}$.

Remark 1. In the sequel we will often need to refer to the highest weight of a representation; to that end we introduce the short notation $\left(\lambda_{1}, \ldots, \lambda_{N}\right)$ for $\left(\lambda_{1}, \ldots, \lambda_{N}, 0, \ldots, 0\right)$ and denote by $\left(\lambda_{1}, \ldots, \lambda_{N}\right)^{\prime}$ the highest weight $\left(\lambda_{1}+\frac{1}{2}, \ldots, \lambda_{N}+\frac{1}{2}, \frac{1}{2}, \ldots, \frac{1}{2}\right)$.

Definition 2. A function $f: \mathbb{R}^{N m} \rightarrow \mathbb{C},\left(\underline{u}_{1}, \ldots, \underline{u}_{N}\right) \mapsto f\left(\underline{u}_{1}, \ldots, \underline{u}_{N}\right)$ is simplicial harmonic if the following conditions are satisfied:

$$
\begin{array}{ll}
\left\langle\underline{\partial}_{i}, \underline{\partial}_{j}\right\rangle f=0, & i, j=1, \ldots, N \\
\left\langle\underline{u}_{i}, \underline{\partial}_{j}\right\rangle f=0, & 1 \leq i<j \leq N .
\end{array}
$$

The vector space of $\mathbb{C}$-valued simplicial harmonic polynomials, $\lambda_{i}$-homogeneous in the variable $\underline{u}_{i}$, will be denoted by $\mathcal{H}_{\lambda_{1}, \ldots, \lambda_{N}}$ (with $\lambda_{1} \geq \ldots \geq \lambda_{N} \geq 0$ from now on).

Definition 3. A function $f: \mathbb{R}^{N m} \rightarrow \mathbb{S},\left(\underline{u}_{1}, \ldots, \underline{u}_{N}\right) \mapsto f\left(\underline{u}_{1}, \ldots, \underline{u}_{N}\right)$ is simplicial monogenic if the following conditions are satisfied:

$$
\begin{array}{ll}
\underline{\partial}_{i} f=0, & i=1, \ldots, N \\
\left\langle\underline{u}_{i}, \underline{\partial}_{j}\right\rangle f=0, & 1 \leq i<j \leq N .
\end{array}
$$

The vector space of $\mathbb{S}$-valued simplicial monogenic polynomials, $\lambda_{i}$-homogeneous in the variable $\underline{u}_{i}$, will be denoted by $\mathcal{S}_{\lambda_{1}, \ldots, \lambda_{N}}$ (with $\lambda_{1} \geq \ldots \geq \lambda_{N} \geq 0$ from now on).

Remark 2. It is clear that if a function is simplicial monogenic in an open region $\Omega$ of $\mathbb{R}^{N m}$, then each of its scalar components is simplicial harmonic in $\Omega$, or in other words: $\mathcal{S}_{\lambda_{1}, \ldots, \lambda_{N}} \subset \mathcal{H}_{\lambda_{1}, \ldots, \lambda_{N}} \otimes \mathbb{S}$.

Remark 3. The second condition in Definition 2 (respectively 3) implies that an arbitrary polynomial $p_{\lambda_{1}, \ldots, \lambda_{N}} \in \mathcal{H}_{\lambda_{1}, \ldots, \lambda_{N}}$ (respectively $\mathcal{S}_{\lambda_{1}, \ldots, \lambda_{N}}$ ) can be identified with a $\mathbb{C}$-valued 
(resp. S-valued) polynomial $f$ depending only of a number of specific wedge products of the vector variables:

$$
p_{\lambda_{1}, \ldots, \lambda_{N}}\left(\underline{u}_{1}, \underline{u}_{2}, \ldots, \underline{u}_{N}\right)=f\left(\underline{u}_{1}, \underline{u}_{1} \wedge \underline{u}_{2}, \underline{u}_{1} \wedge \underline{u}_{2} \wedge \underline{u}_{3}, \ldots, \underline{u}_{1} \wedge \underline{u}_{2} \wedge \ldots \wedge \underline{u}_{N}\right) .
$$

For details we refer to [10], where it is also shown that the space $\mathcal{H}_{\lambda_{1}, \ldots, \lambda_{N}}$ corresponds to the irreducible $\operatorname{Spin}(m)$-module with highest weight $\left(\lambda_{1}, \ldots, \lambda_{N}\right)$, with respect to the regular representation $H$ on $\mathbb{C}$-valued simplicial harmonic polynomials given by

$$
H(s) f\left(\underline{u}_{1}, \underline{u}_{1} \wedge \underline{u}_{2}, \ldots, \underline{u}_{1} \wedge \ldots \wedge \underline{u}_{N}\right)=f\left(\bar{s} \underline{u}_{1} s, \bar{s} \underline{u}_{1} \wedge \underline{u}_{2} s, \ldots, \bar{s} \underline{u}_{1} \wedge \ldots \wedge \underline{u}_{N} s\right),
$$

where $s \in \operatorname{Spin}(m)$. With respect to the regular representation $L$ on $\mathbb{S}$-valued simpicial monogenic polynomials, i.e.

$$
L(s) f\left(\underline{u}_{1}, \underline{u}_{1} \wedge \underline{u}_{2}, \ldots, \underline{u}_{1} \wedge \ldots \wedge \underline{u}_{N}\right)=s f\left(\bar{s} \underline{u}_{1} s, \bar{s} \underline{u}_{1} \wedge \underline{u}_{2} s, \ldots, \bar{s} \underline{u}_{1} \wedge \ldots \wedge \underline{u}_{N} s\right),
$$

the space $\mathcal{S}_{\lambda_{1}, \ldots, \lambda_{N}}$ defines a model for the irreducible (finite-dimensional) $\operatorname{Spin}(m)$-module with highest weight $\left(\lambda_{1}, \ldots, \lambda_{N}\right)^{\prime}$.

Remark 4. As opposed to the one-variable case, the extra conditions in the definition of simplicial monogenic polynomials, involving the operators $\left\langle\underline{u}_{i}, \underline{\partial}_{j}\right\rangle$, are needed in order to obtain an irreducible module for Spin $(m)$. For example, $\mathcal{M}_{k}$ is an irreducible module, while $\mathcal{M}_{\lambda_{1}, \lambda_{2}}:=\left\{f: \mathbb{R}^{2 m} \rightarrow \mathbb{S} \mid \underline{\partial}_{1} f=\underline{\partial}_{2} f=0\right\}$ can be decomposed into irreducible modules, see e.g. [7], by means of

$$
\mathcal{M}_{\lambda_{1}, \lambda_{2}}=\bigoplus_{j=0}^{\lambda_{1}-\lambda_{2}}\left\langle\underline{u}_{2}, \underline{\partial}_{1}\right\rangle^{j} \mathcal{S}_{\lambda_{1}+j, \lambda_{2}-j} .
$$

From now on we take $N=2$ and $\left(\underline{u}_{1}, \underline{u}_{2}\right)=(\underline{u}, \underline{v})$ in Definitions 2 and 3. Our object of interest is the elliptic, $\operatorname{Spin}(m)$-invariant, first-order differential operator

$$
\begin{aligned}
\mathcal{Q}_{k, l}: \mathcal{C}^{\infty}\left(\mathbb{R}^{m}, \mathcal{S}_{k, l}\right) & \rightarrow \mathcal{C}^{\infty}\left(\mathbb{R}^{m}, \mathcal{S}_{k, l}\right) \\
f(\underline{x} ; \underline{u}, \underline{v}) & \mapsto \mathcal{Q}_{k, l} f(\underline{x} ; \underline{u}, \underline{v}) .
\end{aligned}
$$

This higher spin Dirac operator $\mathcal{Q}_{k, l}$ was already constructed in [12] following a pragmatic approach. In this paper we will use techniques from representation theory, which will ease the generalization to the most general case, and describe its polynomial solutions.

\section{Refined Fischer decomposition for simplicial monogenic polynomials}

We proceed as follows for the construction of the higher spin Dirac operator $\mathcal{Q}_{k, l}$. Let $V$ be a representation of $\operatorname{Spin}(m)$ or its Lie algebra $\mathfrak{s o}(m)$. Denote by $\Gamma_{\lambda}$ the finite-dimensional irreducible representation with highest weight $\lambda$. The multiplicity of $\Gamma_{\lambda}$ in $V$ is denoted $m_{\lambda}(V)$ and the multiplicity of an arbitrary weight $\mu$ in $\Gamma_{\lambda}$ is denoted $n_{\mu}\left(\Gamma_{\lambda}\right)$. The following well-known result will be used (for the proof, we refer to e.g. [16]): 
Proposition 1. If $\nu$ is a dominant integral weight such that $m_{\nu}\left(\Gamma_{\lambda} \otimes \Gamma_{\mu}\right)>0$, then there is a weight $\mu^{\prime}$ of $\Gamma_{\mu}$ such that $\nu=\lambda+\mu^{\prime}$ and $m_{\nu}\left(\Gamma_{\lambda} \otimes \Gamma_{\mu}\right) \leq n_{\lambda-\nu}\left(\Gamma_{\mu}\right)$.

One can then also prove the following theorem.

Theorem 1. For any pair of integers $k \geq l \geq 0$ with $k>0$, one has

$$
(k, l) \otimes(0)^{\prime}=(k, l)^{\prime} \oplus\left(1-\delta_{l, 0}\right)(k, l-1)^{\prime} \oplus\left(1-\delta_{k, l}\right)(k-1, l)^{\prime} \oplus\left(1-\delta_{l, 0}\right)(k-1, l-1)^{\prime} .
$$

Proof. Take $\lambda=(k, l), \mu=(0)^{\prime}$ the highest weight for $\mathbb{S}$ and $\nu$ a dominant integral weight such that $m_{\nu}\left(\mathcal{H}_{k, l} \otimes \mathbb{S}\right)>0$. Then, by Proposition 1 , there is a weight $s$ of $\mathbb{S}$ such that $\nu=\lambda+s$ and $m_{\nu}\left(\mathcal{H}_{k, l} \otimes \mathbb{S}\right) \leq n_{s}(\mathbb{S})=1$. The possible weights $\nu$ are given by

$$
\nu=\left(k \pm \frac{1}{2}, l \pm \frac{1}{2}, \pm \frac{1}{2}, \ldots, \pm \frac{1}{2}\right) .
$$

As $\nu$ has to be a dominant integral weight, we only have to deal with the following cases: $\nu=(k, l)^{\prime}, \nu=(k-1, l)^{\prime}$, if $k>l$, and $\nu=(k, l-1)^{\prime}, \nu=(k-1, l-1)^{\prime}$, if $k \geq l>0$. The representations corresponding to these highest weights appear exactly once in $\mathcal{H}_{k, l} \otimes \mathbb{S}$. We show this explicitly for $\nu=(k, l)^{\prime}$ (the other cases being treated similarly). Let $\delta=\left(n-\frac{1}{2}, n-\frac{3}{2}, \ldots, \frac{3}{2}, \frac{1}{2}\right)$ be half the sum of the positive roots and $\mathcal{W}$ the Weyl group. Using Klymik's formula, see e.g. [14], we find

$$
m_{\nu}\left(\mathcal{H}_{k, l} \otimes \mathbb{S}\right)=\sum_{w \in \mathcal{W}} \operatorname{sgn}(w) n_{\nu+\delta-w(\lambda+\delta)}(\mathbb{S})=n_{\nu+\delta-\mathbf{1}(\lambda+\delta)}(\mathbb{S})=n_{\left(\frac{1}{2}, \ldots, \frac{1}{2}\right)}(\mathbb{S})=1 .
$$

This follows from the fact that $w=\mathbf{1} \in \mathcal{W}$ is the only element leading to a non-trivial result in the summation. Indeed, the action of $\mathcal{W}$ changes the sign of the components $\lambda_{i}$ of the weight $\left(\lambda_{1}, \ldots, \lambda_{n}\right)$. In order to satisfy $\lambda_{1} \geq \ldots \geq \lambda_{n}$, only the trivial action remains. This proves the claim.

In case $l=0$, the previous result encodes the Fischer decomposition for spinor-valued harmonic polynomials: $\mathcal{H}_{k} \otimes \mathbb{S}=\mathcal{M}_{k} \oplus \underline{u} \mathcal{M}_{k-1}$. This result is well-known in Clifford analysis and states that any $\mathbb{S}$-valued harmonic homogeneous polynomial $H_{k}$ of degree $k$ in the vector variable $\underline{u}$ can be decomposed in terms of two monogenic homogeneous polynomials

$$
H_{k}=M_{k}+\underline{u} M_{k-1},
$$

with $M_{\lambda} \in \mathcal{M}_{\lambda}$. The factor $\underline{u}$ in this formula is called an embedding factor: it realizes an isomorphic copy of the irreducible module $\mathcal{M}_{k-1}$ inside the tensor product $\mathcal{H}_{k} \otimes \mathbb{S}$. Moreover, one can show that these polynomials are explicitly given by

$$
\begin{aligned}
M_{k-1} & =-\frac{1}{m+2 k-2} \underline{\partial}_{u} H_{k} \\
M_{k} & =\left(1+\frac{\underline{u} \underline{\partial}_{u}}{m+2 k-2}\right) H_{k} .
\end{aligned}
$$


We will now generalize this result to the present setting $k \geq l \geq 0$. Theorem 1 tells us how the space of $\mathbb{S}$-valued simplicial harmonic polynomials $\mathcal{H}_{k, l} \otimes \mathbb{S}$ decomposes into irreducible summands. It implies the existence of certain maps which embed each of the spaces $\mathcal{S}_{k, l}, \mathcal{S}_{k-1, l}, \mathcal{S}_{k, l-1}$ and $\mathcal{S}_{k-1, l-1}$ (for appropriate $k$ and $l$ ) into the space $\mathcal{H}_{k, l} \otimes \mathbb{S}$. To ensure that these embedding maps are indeed morphisms realizing an isomorphic copy of the spaces of simplicial monogenics inside the space $\mathcal{H}_{k, l} \otimes \mathbb{S}$, we have to check, next to the homogeneity conditions, whether the conditions in Definition 2 are satisfied. Clearly, $\mathcal{S}_{k, l} \hookrightarrow \mathcal{H}_{k, l} \otimes \mathbb{S}$ is the trivial embedding. Also, it is easily verified that

$$
\underline{u}: \mathcal{S}_{k-1, l} \hookrightarrow \mathcal{H}_{k, l} \otimes \mathbb{S} .
$$

In order to embed the space $\mathcal{S}_{k, l-1}$ into the tensor product $\mathcal{H}_{k, l} \otimes \mathbb{S}$, it seems obvious to start from the basic invariant $\underline{v}$, as we need an embedding map of degree $(0,1)$ in $(\underline{u}, \underline{v})$, but this approach fails since $\left\langle\underline{u}, \underline{\partial}_{v}\right\rangle\left[\underline{v} \mathcal{S}_{k, l-1}\right]=\underline{u} \mathcal{S}_{k, l-1} \neq 0$. In order to obtain the required embedding map, it suffices to project onto the kernel of the operator $\left\langle\underline{u}, \underline{\partial}_{v}\right\rangle$, which can be done by fixing $c_{1}$ in the following expression:

$$
\underline{v}-c_{1} \underline{u}\left\langle\underline{v}, \underline{\partial}_{u}\right\rangle: \mathcal{S}_{k, l-1} \hookrightarrow \mathcal{H}_{k, l} \otimes \mathbb{S} .
$$

For $c_{1}=\frac{1}{k-l+1}$ all conditions in Definition 2 are indeed satisfied. Similarly, the last embedding map can be found as a suitable projection of a linear combination of $\underline{u} \underline{v}$ and $\underline{v} \underline{u}$, and is given by

$$
\langle\underline{v}, \underline{u}\rangle-c_{2} \underline{v} \underline{u}-c_{3}\langle\underline{u}, \underline{u}\rangle\left\langle\underline{v}, \underline{\partial}_{u}\right\rangle: \mathcal{S}_{k-1, l-1} \hookrightarrow \mathcal{H}_{k, l} \otimes \mathbb{S}
$$

with $c_{2}=-\frac{m+k+l-4}{m+2 k-4}$ and $c_{3}=\frac{1}{m+2 k-4}$. This can be summarized as follows:

Proposition 2. For any pair of integers $k \geq l \geq 0$ with $k>0$, one has

$$
\mathcal{H}_{k, l} \otimes \mathbb{S}=\mathcal{S}_{k, l} \oplus\left(1-\delta_{l, 0}\right) \nu_{k, l} \mathcal{S}_{k, l-1} \oplus\left(1-\delta_{k, l}\right) \mu_{k, l} \mathcal{S}_{k-1, l} \oplus\left(1-\delta_{l, 0}\right) \kappa_{k, l} \mathcal{S}_{k-1, l-1},
$$

with the embedding maps

$$
\begin{aligned}
\nu_{k, l} & :=\underline{v}-\frac{\underline{u}\left\langle\underline{v}, \underline{\partial}_{u}\right\rangle}{k-l+1}: \mathcal{S}_{k, l-1} \hookrightarrow \mathcal{H}_{k, l} \otimes \mathbb{S} \\
\mu_{k, l} & :=\underline{u}: \mathcal{S}_{k-1, l} \hookrightarrow \mathcal{H}_{k, l} \otimes \mathbb{S} \\
\kappa_{k, l} & :=\langle\underline{v}, \underline{u}\rangle+\frac{m+k+l-4}{m+2 k-4} \underline{v} \underline{u}-\frac{\langle\underline{u}, \underline{u}\rangle\left\langle\underline{v}, \underline{\partial}_{u}\right\rangle}{m+2 k-4}: \mathcal{S}_{k-1, l-1} \hookrightarrow \mathcal{H}_{k, l} \otimes \mathbb{S} .
\end{aligned}
$$

Remark 5. The embedding map $\mu_{k, k}$ clearly does not exist, in view of the dominant weight condition. If $l=0$ the embedding maps $\nu_{k, l}$ and $\kappa_{k, l}$ do not exist neither.

Let $k>l>0$ and suppose $\psi \in \mathcal{H}_{k, l} \otimes \mathbb{S}$. According to Proposition 2, there exists $\psi_{p, q} \in \mathcal{S}_{p, q}$ such that

$$
\psi=\psi_{k, l}+\nu_{k, l} \psi_{k, l-1}+\mu_{k, l} \psi_{k-1, l}+\kappa_{k, l} \psi_{k-1, l-1}
$$


An explicit expression for the projection operators on each of the summands inside $\mathcal{H}_{k, l} \otimes \mathbb{S}$ can then be obtained as follows. First, the action of $\underline{\partial}_{v}$ on (1) annihilates two summands and leads to

$$
\underline{\partial}_{v} \psi=-(m+2 l-4) \psi_{k, l-1}+\left(1-\frac{m+k+l-4}{m+2 k-4}(m+2 l-2)\right) \underline{u} \psi_{k-1, l-1} .
$$

Acting again with $\underline{\partial}_{u}$, we find

$$
\psi_{k-1, l-1}=\frac{(m+2 k-4) \underline{\partial}_{u} \underline{\partial}_{v} \psi}{(m+2 k-2)(m+2 l-4)(m+k+l-3)} .
$$

This gives rise to a projection operator $\pi_{k-1, l-1}$, defined as

$$
\begin{aligned}
\pi_{k-1, l-1}: \mathcal{H}_{k, l} \otimes \mathbb{S} & \rightarrow \mathcal{S}_{k-1, l-1} \\
\psi & \mapsto \frac{(m+2 k-4) \underline{\partial}_{u} \underline{\partial}_{v} \psi}{(m+2 k-2)(m+2 l-4)(m+k+l-3)} .
\end{aligned}
$$

Substituting the expression for $\psi_{k-1, l-1}$ in (2), we find

$$
\psi_{k, l-1}=-\frac{1}{m+2 l-4}\left(1+\frac{\underline{u} \underline{\partial}_{u}}{m+2 k-2}\right) \underline{\partial}_{v} \psi,
$$

which defines a second projection operator $\pi_{k, l-1}$ :

$$
\begin{aligned}
\pi_{k, l-1}: \mathcal{H}_{k, l} \otimes \mathbb{S} & \rightarrow \mathcal{S}_{k, l-1} \\
\psi & \mapsto-\frac{1}{m+2 l-4}\left(1+\frac{\underline{u} \underline{\partial}_{u}}{m+2 k-2}\right) \underline{\partial}_{v} \psi .
\end{aligned}
$$

Finally, using the previous results, the action of $\underline{\partial}_{u}$ on (1) leads to

$$
\begin{aligned}
& \psi_{k-1, l}=-\frac{1}{m+2 k-2}[(\mathbf{1} \\
&\left.+\left(\frac{k-l}{k-l+1}\right) \frac{\underline{v}_{v}}{m+2 l-4}\right) \underline{\partial}_{u} \\
&\left.+\frac{1}{k-l+1}\left(\mathbf{1}+\frac{\underline{u}_{u}}{m+2 l-4}\right)\left\langle\underline{v}, \underline{\partial}_{u}\right\rangle \underline{\partial}_{v}\right] \psi,
\end{aligned}
$$

which defines the third projection operator:

$$
\begin{aligned}
\pi_{k-1, l}: \mathcal{H}_{k, l} \otimes \mathbb{S} & \rightarrow \mathcal{S}_{k-1, l} \\
\psi & \mapsto-\frac{1}{m+2 k-2}\left[\left(\mathbf{1}+\left(\frac{k-l}{k-l+1}\right) \frac{\underline{v}^{\underline{\partial}} \underline{\partial}_{v}}{m+2 l-4}\right) \underline{\partial}_{u}\right. \\
& \left.\quad+\frac{1}{k-l+1}\left(\mathbf{1}+\frac{\underline{u} \underline{\partial}_{u}}{m+2 l-4}\right)\left\langle\underline{v}_{2} \underline{\partial}_{u}\right\rangle \underline{\partial}_{v}\right] \psi
\end{aligned}
$$

The last projection operator on the summand $\mathcal{S}_{k, l}$ is then given by

$$
\pi_{k, l}:=\mathbf{1}-\pi_{k-1, l}-\pi_{k, l-1}-\pi_{k-1, l-1} .
$$

We gather all this information in the following theorem. 
Theorem 2. (Refined Fischer decomposition for simplicial monogenics.)

Each $\mathbb{S}$-valued simplicial harmonic polynomial $H_{k, l}$ in two vector variables can be uniquely decomposed in terms of simplicial monogenic polynomials:

$$
H_{k, l}=M_{k, l}+\nu_{k, l} M_{k, l-1}+\mu_{k, l} M_{k-1, l}+\kappa_{k, l} M_{k-1, l-1},
$$

with the embedding maps defined in Proposition 2 and with

$$
\begin{aligned}
M_{k, l} & =\pi_{k, l}\left(H_{k, l}\right) & M_{k, l-1} & =\pi_{k, l-1}\left(H_{k, l}\right) \\
M_{k-1, l} & =\pi_{k-1, l}\left(H_{k, l}\right) & M_{k-1, l-1} & =\pi_{k-1, l-1}\left(H_{k, l}\right) .
\end{aligned}
$$

Proof. Only the uniqueness has to be addressed, but this can easily be proved.

\section{Construction of the operator $\mathcal{Q}_{k, l}$}

We now use the refined Fischer decomposition of Theorem 2 to construct the higher spin Dirac operator $\mathcal{Q}_{k, l}$. Since the classical Dirac operator $\underline{\partial}_{x}$ can be seen as an endomorphism on $\mathbb{S}$-valued functions, the action of $\underline{\partial}_{x}$ on a $\mathcal{H}_{k, l} \otimes \mathbb{S}$-valued function preserves the values. This gives rise to a collection of invariant operators defined through the following diagram:

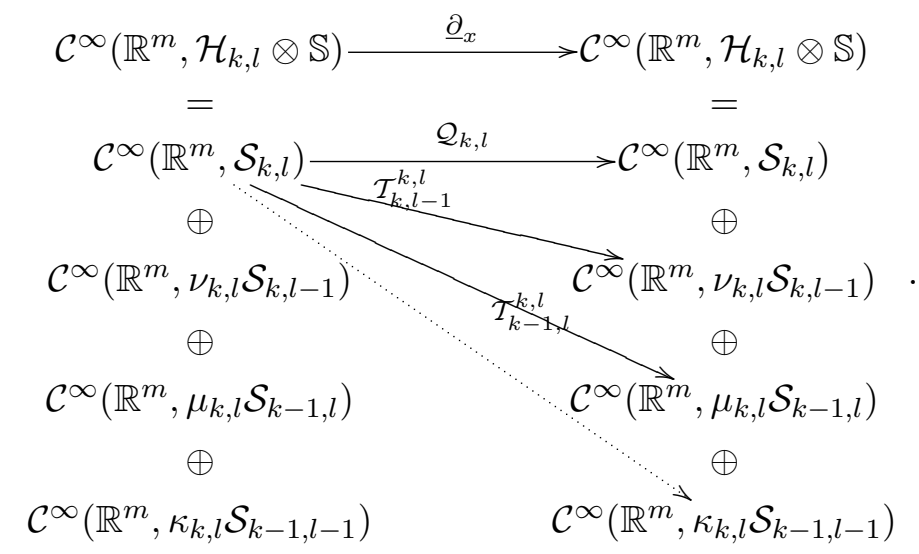

The non-existence of an invariant operator from $\mathcal{C}^{\infty}\left(\mathbb{R}^{m}, \mathcal{S}_{k, l}\right)$ to $\mathcal{C}^{\infty}\left(\mathbb{R}^{m}, \kappa_{k, l} \mathcal{S}_{k-1, l-1}\right)$ (i.e. the dotted arrow in the diagram above) can be proved by means of the construction method of conformally invariant operators using generalized gradients, see e.g. [13, 22]. It essentially follows from the fact that the tensor product $\mathcal{S}_{k, l} \otimes \mathbb{C}^{m}$ does not contain the summand $\mathcal{S}_{k-1, l-1}$. The next lemma shows that this can also be verified through direct calculations in Clifford analysis.

Lemma 1. For every $f \in \mathcal{C}^{\infty}\left(\mathbb{R}^{m}, \mathcal{S}_{k, l}\right)$ one has $\underline{\partial}_{u} \underline{\partial}_{v} \underline{\partial}_{x} f=0$.

Proof. The definition of the Euclidean inner product leads to

$$
\underline{\partial}_{u} \underline{\partial}_{v} \underline{\partial}_{x} f=-2 \underline{\partial}_{u}\left\langle\underline{\partial}_{v}, \underline{\partial}_{x}\right\rangle f-\underline{\partial}_{u} \underline{\partial}_{x} \underline{\partial}_{v} f=0,
$$


since $\underline{\partial}_{u} f=\underline{\partial}_{v} f=0$.

Hence, it follows from (3) that $\pi_{k-1, l-1}\left(\underline{\partial}_{x} f\right) \equiv 0$, for every $f \in \mathcal{C}^{\infty}\left(\mathbb{R}^{m}, \mathcal{S}_{k, l}\right)$. An explicit expression for the operators $\mathcal{Q}_{k, l}, \mathcal{T}_{k, l-1}^{k, l}$ and $\mathcal{T}_{k-1, l}^{k, l}$ in the diagram above is then obtained using results of the previous section.

Definition 4. For all integers $k \geq l \geq 0$ with $k>0$, there are (up to a multiplicative constant) unique invariant first-order differential operators $\mathcal{Q}_{k, l}$ defined by

$$
\mathcal{Q}_{k, l}: \mathcal{C}^{\infty}\left(\mathbb{R}^{m}, \mathcal{S}_{k, l}\right) \rightarrow \mathcal{C}^{\infty}\left(\mathbb{R}^{m}, \mathcal{S}_{k, l}\right): f \mapsto \pi_{k, l}\left(\underline{\partial}_{x} f\right),
$$

or explicitly,

$$
\mathcal{Q}_{k, l} f=\left[\mathbf{1}+\frac{\underline{u} \underline{\partial}_{u}}{m+2 k-2}+\frac{\underline{v} \underline{\partial}_{v}}{m+2 l-4}-2 \frac{\underline{u}\left\langle\underline{v}, \underline{\partial}_{u}\right\rangle \underline{\partial}_{v}}{(m+2 k-2)(m+2 l-4)}\right] \underline{\partial}_{x} f .
$$

In case $k=l>0$, the operators reduce to

$$
\mathcal{Q}_{k, k} f=\left[\mathbf{1}+\frac{\left(\underline{v}-\underline{u}\left\langle\underline{v}, \underline{\partial}_{u}\right\rangle\right) \underline{\partial}_{v}}{m+2 k-4}\right] \underline{\partial}_{x} f .
$$

Remark 6. In case $l=0$, we find the Rarita-Schwinger operators $\mathcal{R}_{k}$, as defined in [7]:

$$
\mathcal{Q}_{k, 0}=\mathcal{R}_{k}=\left(1+\frac{\underline{u}_{u}}{m+2 k-2}\right) \underline{\partial}_{x}
$$

The ellipticity of this operator $\mathcal{Q}_{k, l}$ follows e.g. from [5], and the $\operatorname{Spin}(m)$-invariance can be expressed through the following commutative diagram:

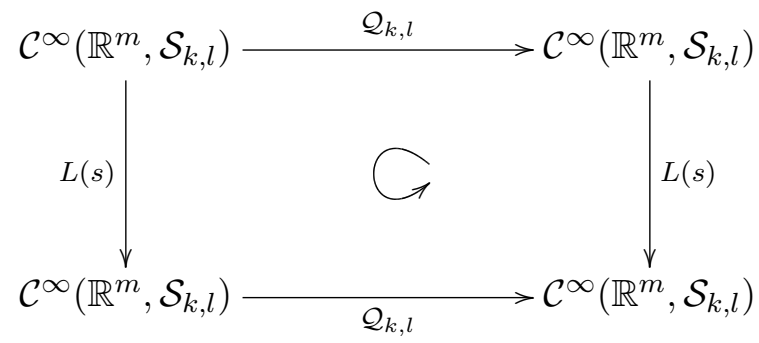

with $L(s)(f(\underline{u}, \underline{v}))=s f(\bar{s} \underline{u} s, \bar{s} \underline{v} s)$ the natural action of $\operatorname{Spin}(m)$ on higher spin fields.

Similar calculations lead to the so-called dual twistor operators, which are visualized as the diagonal arrows in the diagram above. We adopt the convention that each operator of twistor-type will be denoted by means of the letter $\mathcal{T}$, together with upper and lower indices. The upper indices denote the highest weight of the source space, whereas the lower indices denote the highest weight of the target space (discarding the half-integers).

Definition 5. For all integers $k>l \geq 0$, the dual twistor operators $\mathcal{T}_{k-1, l}^{k, l}$ are defined as the unique invariant first-order differential operators

$$
\mathcal{T}_{k-1, l}^{k, l}: \mathcal{C}^{\infty}\left(\mathbb{R}^{m}, \mathcal{S}_{k, l}\right) \rightarrow \mathcal{C}^{\infty}\left(\mathbb{R}^{m}, \mu_{k, l} \mathcal{S}_{k-1, l}\right): f \mapsto \mu_{k, l} \pi_{k-1, l}\left(\underline{\partial}_{x} f\right)
$$


or explicitly, hereby taking Lemma 1 into account:

$$
\mathcal{T}_{k-1, l}^{k, l} f=\mu_{k, l}\left[\frac{2}{m+2 k-2}\left(\left\langle\underline{\partial}_{u}, \underline{\partial}_{x}\right\rangle+\frac{\left\langle\underline{v}, \underline{\partial}_{u}\right\rangle\left\langle\underline{\partial}_{v}, \underline{\partial}_{x}\right\rangle}{k-l+1}\right) f\right] .
$$

In case $k=l>0$, these operators do not exist.

Definition 6. For all integers $k>l>0$, the dual twistor operators $\mathcal{T}_{k, l-1}^{k, l}$ are defined as the unique invariant first-order differential operators

$$
\mathcal{T}_{k, l-1}^{k, l}: \mathcal{C}^{\infty}\left(\mathbb{R}^{m}, \mathcal{S}_{k, l}\right) \rightarrow \mathcal{C}^{\infty}\left(\mathbb{R}^{m}, \nu_{k, l} \mathcal{S}_{k, l-1}\right): f \mapsto \nu_{k, l} \pi_{k, l-1}\left(\underline{\partial}_{x} f\right)
$$

or explicitly, again taking Lemma 1 into account:

$$
\mathcal{T}_{k, l-1}^{k, l} f=\nu_{k, l}\left(\frac{2}{m+2 l-4}\left\langle\underline{\partial}_{v}, \underline{\partial}_{x}\right\rangle f\right) .
$$

In case $k=l>0$, these operators reduce to

$$
\mathcal{T}_{k, k-1}^{k, k} f=\frac{2}{m+2 k-4}\left(\underline{v}-\underline{u}\left\langle\underline{v}, \underline{\partial}_{u}\right\rangle\right)\left\langle\underline{\partial}_{v}, \underline{\partial}_{x}\right\rangle f .
$$

Note that these operators are called dual, because there exist also twistor operators acting in the opposite direction; these ones are given below, but they will not be explicitly used in this paper.

Remark 7. The twistor operators $\mathcal{T}_{k, l}^{k-1, l}$ and $\mathcal{T}_{k, l}^{k, l-1}$ are defined as

$$
\mathcal{T}_{k, l}^{k-1, l}: \mathcal{C}^{\infty}\left(\mathbb{R}^{m}, \mu_{k, l} \mathcal{S}_{k-1, l}\right) \rightarrow \mathcal{C}^{\infty}\left(\mathbb{R}^{m}, \mathcal{S}_{k, l}\right): \mu_{k, l} f \mapsto \pi_{k, l}\left(\underline{\partial}_{x} \mu_{k, l} f\right)
$$

and

$$
\mathcal{T}_{k, l}^{k, l-1}: \mathcal{C}^{\infty}\left(\mathbb{R}^{m}, \nu_{k, l} \mathcal{S}_{k, l-1}\right) \rightarrow \mathcal{C}^{\infty}\left(\mathbb{R}^{m}, \mathcal{S}_{k, l}\right): \nu_{k, l} f \mapsto \pi_{k, l}\left(\underline{\partial}_{x} \nu_{k, l} f\right) .
$$

Remark 8. We introduce the following short notations for the dual twistor operators without the embedding factor:

$$
\begin{aligned}
& \mathbb{T}_{k, l-1}^{k, l}:=\left(\nu_{k, l}\right)^{-1} \mathcal{T}_{k, l-1}^{k, l}=\left\langle\underline{\partial}_{v}, \underline{\partial}_{x}\right\rangle \\
& \mathbb{T}_{k-1, l}^{k, l}:=\left(\mu_{k, l}\right)^{-1} \mathcal{T}_{k-1, l}^{k, l}=\left\langle\underline{\partial}_{u}, \underline{\partial}_{x}\right\rangle+\frac{\left\langle\underline{v}, \underline{\partial}_{u}\right\rangle\left\langle\underline{\partial}_{v}, \underline{\partial}_{x}\right\rangle}{k-l+1} .
\end{aligned}
$$

\section{Constructing polynomial null solutions}

As in any function theory linked to a differential operator, a crucial piece of knowledge is the full description of its polynomial null solutions. This will be the subject of this section. Note that in this respect, higher spin Dirac operators behave completely different from the classical Dirac operator: the spaces of polynomial null solutions will no longer be irreducible as a $\operatorname{Spin}(m)$-module, and a typical problem is to decompose polynomial 
kernel spaces for higher spin Dirac operators into irreducibles.

Let us denote by $\operatorname{Ker}_{h} \mathcal{D}$ the vector space of $h$-homogeneous polynomial null solutions of the (linear differential) operator $\mathcal{D}$. As a function $f$ belongs to $\operatorname{Ker}_{h} \mathcal{Q}_{k, l}$ if and only if it satisfies $\pi_{k, l}\left(\underline{\partial}_{x} f\right)=0$, there are two possibilities to satisfy this condition. This gives rise to two types of homogeneous polynomial null solutions $f$ for $\mathcal{Q}_{k, l}$ : either $\underline{\partial}_{x} f=0$ (called type A solutions) or $\underline{\partial}_{x} f \neq 0$ but $\pi_{k, l}\left(\underline{\partial}_{x} f\right)=0$ (called type B solutions). We will now treat each of these possibilities in detail.

\subsection{Solutions of Type A}

For all integers $h \geq k \geq l>0$, define $\mathcal{P}_{h, k, l}(\mathbb{S})$ to be the space of $\mathbb{S}$-valued polynomials in three vector variables $(\underline{x}, \underline{u}, \underline{v})$, homogeneous of degree $h, k$ and $l$ in $\underline{x}, \underline{u}$ and $\underline{v}$ respectively. Denote the subspace of triple monogenics by

$$
\mathcal{M}_{h, k, l}=\left\{f \in \mathcal{P}_{h, k, l}(\mathbb{S}) \mid \underline{\partial}_{x} f=\underline{\partial}_{u} f=\underline{\partial}_{v} f=0\right\} .
$$

This vector space is highly reducible with respect to the action of $\operatorname{Spin}(m)$, and in [4] we have determined the decomposition of this space in terms of irreducible $\operatorname{Spin}(m)$-modules, making use of the fact that each vector space $\mathcal{S}_{p, q, r}$ can be seen as a highest weight vector for the algebra $\mathfrak{g l}_{3}$, with positive root vectors $\left\{\left\langle\underline{x}, \underline{\partial}_{u}\right\rangle,\left\langle\underline{x}, \underline{\partial}_{v}\right\rangle,\left\langle\underline{u}, \underline{\partial}_{v}\right\rangle\right\}$. The vector space $\mathcal{M}_{h, k, l}^{s}=\mathcal{M}_{h, k, l} \cap \operatorname{Ker}\left\langle\underline{u}, \underline{\partial}_{v}\right\rangle$, or more explicitly $\mathcal{M}_{h, k, l}^{s}=\left\{f \in \mathcal{M}_{h, k, l} \mid\left\langle\underline{u}, \underline{\partial}_{v}\right\rangle f=0\right\}$, is, by construction, precisely the space of $h$-homogeneous solutions for $\mathcal{Q}_{k, l}$ of type A. The decomposition of this space into irreducible spaces for $\operatorname{Spin}(m)$ was also determined in [4], using branching rules from $\mathfrak{g l}_{3}$ to $\mathfrak{g l}_{2}$. Using the so-called raising and lowering operators $\left\langle\underline{u}, \underline{\partial}_{x}\right\rangle$ and $\left\langle\underline{v}, \underline{\partial}_{x}\right\rangle\left(\mathbb{E}_{u}-\mathbb{E}_{v}\right)-\left\langle\underline{u}, \underline{\partial}_{x}\right\rangle\left\langle\underline{v}, \underline{\partial}_{u}\right\rangle$, which were studied in the much broader setting of transvector algebras and weight bases for Lie algebras in e.g. [18], we were able to prove that for $k \geq l$,

$$
\mathcal{M}_{h, k, l}^{s}=\bigoplus_{i=0}^{k-l} \bigoplus_{j=0}^{l}\left\langle\underline{u}, \underline{\partial}_{x}\right\rangle^{i}\left[\left\langle\underline{v}, \underline{\partial}_{x}\right\rangle\left(\mathbb{E}_{u}-\mathbb{E}_{v}\right)-\left\langle\underline{u}, \underline{\partial}_{x}\right\rangle\left\langle\underline{v}, \underline{\partial}_{u}\right\rangle\right]^{j} \mathcal{S}_{h+i+j, k-i, l-j} .
$$

To lighten the notation, we will often omit these (commuting) embedding factors and denote the irreducible modules in these decompositions by their highest weights only:

$$
\mathcal{M}_{h, k, l}^{s}=\bigoplus_{i=0}^{k-l} \bigoplus_{j=0}^{l}(h+i+j, k-i, l-j)^{\prime} .
$$

In the special case where $l=0$, we reobtain the results mentioned earlier in Remark 4:

$$
\mathcal{M}_{h, k}:=\mathcal{M}_{h, k, 0}^{s}=\bigoplus_{i=0}^{k}(h+i, k-i)^{\prime} .
$$

Remark 9. Every irreducible module in $\mathcal{M}_{h, k, l}^{s}$ appears with multiplicity one, which is a general fact for branching rules from $\mathfrak{g l}_{n}$ to $\mathfrak{g l}_{n-1}$. A necessary condition for a module $\mathcal{S}_{p, q, r}$ to be, up to an isomorphic copy, in the decomposition of $\mathcal{M}_{h, k, l}^{s}$ is $p+q+r=h+k+l$. 


\subsection{Solutions of Type B}

A different approach is required to describe the type $\mathrm{B}$ solutions. It is instructive to recall the Rarita-Schwinger case. Let $f(\underline{x} ; \underline{u})$ be a polynomial in $\mathcal{C}^{\infty}\left(\mathbb{R}^{m}, \mathcal{M}_{k}\right)$, homogeneous of degree $h \geq k$ in the vector variable $\underline{x}$. In [7], the following equivalence was proved:

$$
f \in \operatorname{Ker}_{h} \mathcal{R}_{k} \Leftrightarrow\left\{\begin{array}{l}
\underline{\partial}_{x} f=\underline{u} g \\
\underline{\partial}_{u} f=0
\end{array},\right.
$$

with $g \in \operatorname{Ker}_{h-1} \mathcal{R}_{k-1}$. In order for this inhomogeneous system of equations (involving the classical Dirac operator) to have a solution $f$, certain conditions on $g$ must be satisfied. The necessary and sufficient conditions (called compatibility conditions for short) under which an inhomogeneous system in several Dirac operators has a solution, were thoroughly studied in [9]. Referring to [7,9] for details, the compatibility conditions for the existence of a solution for the system above are $\Delta_{u}(\underline{u} g)=0$ and $\underline{\partial}_{u} \underline{\partial}_{x}(\underline{u} g)=0$. The first condition is equivalent with the monogeneity of $g$ in the variable $\underline{u}$, i.e. $g \in \mathcal{C}^{\infty}\left(\mathbb{R}^{m}, \mathcal{M}_{k-1}\right)$, whereas the second compatibility condition is equivalent to $g \in \mathrm{Ker}_{h-1} \mathcal{R}_{k-1}$. In other words: these compatibility conditions signify that the kernel space for the operator $\mathcal{R}_{k-1}$ can be embedded into the kernel space for $\mathcal{R}_{k}$, using certain inversion operators which were described in [7]. Type B solutions for $\mathcal{R}_{k}$ are thus equivalent with elements of $\operatorname{Ker}_{h-1} \mathcal{R}_{k-1}$; their structure may thus be described through an inductive procedure.

In the case of the operator $\mathcal{Q}_{k, l}$, we have the equivalence

$$
\mathcal{Q}_{k, l} f=0 \quad \Leftrightarrow \quad \underline{\partial}_{x} f=\mu_{k, l} \frac{2}{m+2 k-2} \mathbb{T}_{k-1, l}^{k, l} f+\nu_{k, l} \frac{2}{m+2 l-4} \mathbb{T}_{k, l-1}^{k, l} f .
$$

Moreover, we also have the following results, which can be proved by direct calculations:

Proposition 3. For any couple of integers $k>l>0$, resp. $k \geq l>0$, and for any function $f \in \mathcal{C}^{\infty}\left(\mathbb{R}^{m}, \mathcal{S}_{k, l}\right)$, one has: $\pi_{k-1, l}\left(\underline{\partial}_{x} \mathcal{T}_{k, l-1}^{k, l} f\right)=0$, resp. $\pi_{k, l-1}\left(\underline{\partial}_{x} \mathcal{T}_{k-1, l}^{k, l} f\right)=0$.

This means that non-trivial maps $\mathcal{C}^{\infty}\left(\mathbb{R}^{m}, \mu_{k, l} \mathcal{S}_{k-1, l}\right) \rightarrow \mathcal{C}^{\infty}\left(\mathbb{R}^{m}, \nu_{k, l} \mathcal{S}_{k, l-1}\right)$ (and vice versa) do not exist. They are visualized by the dotted lines in the diagram below, where the double action of the Dirac operator on $\mathcal{C}^{\infty}\left(\mathbb{R}^{m}, \mathcal{S}_{k, l}\right)$ is considered.

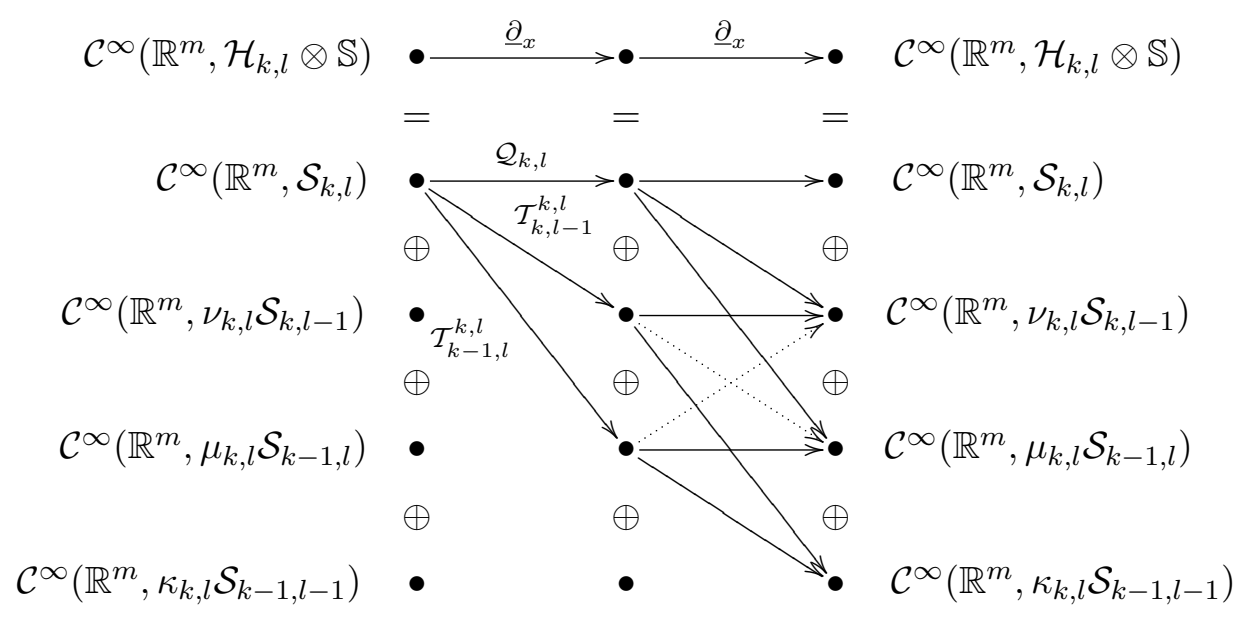


Proposition 4. Let $f \in \operatorname{Ker}_{h} \mathcal{Q}_{k, l}$.

(i) If $k \geq l>0$, then $\mathbb{T}_{k, l-1}^{k, l} f \in \operatorname{Ker}_{h-1} \mathcal{Q}_{k, l-1}$.

(ii) If $k>l \geq 0$, then $\mathbb{T}_{k-1, l}^{k, l} \in \operatorname{Ker}_{h-1} \mathcal{Q}_{k-1, l}$.

Proof. Again, a straightforward calculation leads to the desired result. Let $c_{1}=m+2 k-2$ and $c_{2}=m+2 l-4$. For every $f \in \operatorname{Ker}_{h} \mathcal{Q}_{k, l}$, we have

$$
\begin{aligned}
\left\langle\underline{\partial}_{v}, \underline{\partial}_{x}\right\rangle \mathcal{Q}_{k, l} f=0 & \Leftrightarrow\left\langle\underline{\partial}_{v}, \underline{\partial}_{x}\right\rangle\left[c_{1} c_{2}+c_{2} \underline{u}_{\underline{\partial}}+c_{1} \underline{v} \underline{\partial}_{v}-2 \underline{u}\left\langle\underline{v}, \underline{\partial}_{u}\right\rangle \underline{\partial}_{v}\right] \underline{\partial}_{x} f=0 \\
& \Leftrightarrow \mathcal{Q}_{k, l-1}\left\langle\underline{\partial}_{v}, \underline{\partial}_{x}\right\rangle f=0 .
\end{aligned}
$$

This may also be proved by considering the double action of the Dirac operator. Since $\underline{\partial}_{x}^{2}$ is scalar, the following implication obviously holds:

$$
f \in \mathcal{C}^{\infty}\left(\mathbb{R}^{m}, \mathcal{S}_{k, l}\right) \Rightarrow \underline{\partial}_{x}^{2} f \in \mathcal{C}^{\infty}\left(\mathbb{R}^{m}, \mathcal{S}_{k, l}\right) .
$$

Therefore, the projection on each of the other summands in the decomposition of $\mathcal{H}_{k, l} \otimes \mathbb{S}$ is zero. In particular, we have that $\pi_{k, l-1}\left(\underline{\partial}_{x}^{2} f\right)=0$, which in combination with Proposition 3 leads to the following identity (up to a suitable constant):

$$
\mathcal{T}_{k, l-1}^{k, l} \mathcal{Q}_{k, l}+\nu_{k, l} \mathcal{Q}_{k, l-1}\left(\nu_{k, l}\right)^{-1} \mathcal{T}_{k, l-1}^{k, l}=0 .
$$

This can be visualized by the parallelogram formed by the double arrows in the diagram below:

$$
\begin{aligned}
& \mathcal{C}^{\infty}\left(\mathbb{R}^{m}, \mathcal{H}_{k, l} \otimes \mathbb{S}\right) \stackrel{\underline{\partial}_{x} \longrightarrow \bullet \underline{\partial}_{x}}{\underline{\partial}_{x}^{2}} \gg \bullet \quad \mathcal{C}^{\infty}\left(\mathbb{R}^{m}, \mathcal{H}_{k, l} \otimes \mathbb{S}\right) \\
& =\quad=\quad= \\
& \mathcal{C}^{\infty}\left(\mathbb{R}^{m}, \mathcal{S}_{k, l}\right) \bullet \frac{\mathcal{Q}_{k, l}}{\underset{\mathcal{T}^{k, l}, 1}{\longrightarrow} \bullet} \quad \bullet \mathcal{C}^{\infty}\left(\mathbb{R}^{m}, \mathcal{S}_{k, l}\right) \\
& \mathcal{C}^{\infty}\left(\mathbb{R}^{m}, \nu_{k, l} \mathcal{S}_{k, l-1}\right) \stackrel{\oplus}{\bullet}{ }_{\mathcal{T}_{k-1, l}^{k, l} \backslash} \mathcal{C}_{\bullet}^{\infty}\left(\mathbb{R}^{m}, \nu_{k, l} \mathcal{S}_{k, l-1}\right)
\end{aligned}
$$

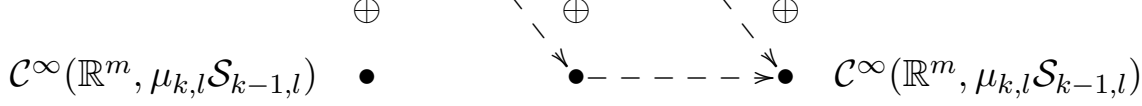

$$
\begin{aligned}
& \oplus \quad \oplus \quad \oplus \\
& \mathcal{C}^{\infty}\left(\mathbb{R}^{m}, \kappa_{k, l} \mathcal{S}_{k-1, l-1}\right) \quad \bullet \quad \bullet \quad \quad \quad \mathcal{C}^{\infty}\left(\mathbb{R}^{m}, \kappa_{k, l} \mathcal{S}_{k-1, l-1}\right)
\end{aligned}
$$

For $f \in \operatorname{Ker}_{h} \mathcal{Q}_{k, l}$, the identity (9) reduces to $\nu_{k, l} \mathcal{Q}_{k, l-1} \mathbb{T}_{k, l-1}^{k, l} f=0$, proving the first statement. The calculations for proving the second statement are somewhat more technical and involved, but using operator identities in Clifford analysis one can verify that

$$
\begin{aligned}
& \left\langle\underline{\partial}_{u}, \underline{\partial}_{x}\right\rangle \mathcal{Q}_{k, l} f+\frac{2}{c_{1}-c_{2}}\left\langle\underline{v}_{u} \underline{\partial}_{u}\right\rangle\left\langle\underline{\partial}_{v}, \underline{\partial}_{x}\right\rangle \mathcal{Q}_{k, l} f=0 \\
& \quad \Rightarrow \quad \mathcal{Q}_{k-1, l}\left(\left\langle\underline{\partial}_{u}, \underline{\partial}_{x}\right\rangle+\frac{\left\langle\underline{v}, \underline{\partial}_{u}\right\rangle\left\langle\underline{\partial}_{v}, \underline{\partial}_{x}\right\rangle}{k-l+1}\right) f=0,
\end{aligned}
$$


which leads to the desired statement. Invoking once more Proposition 3, this can also be proved by considering the parallelogram formed by the dashed lines in the diagram above, leading to the identity

$$
\mathcal{T}_{k-1, l}^{k, l} \mathcal{Q}_{k, l}+\mu_{k, l} \mathcal{Q}_{k-1, l}\left(\mu_{k, l}\right)^{-1} \mathcal{T}_{k-1, l}^{k, l}=0,
$$

For $f \in \operatorname{Ker}_{h} \mathcal{Q}_{k, l}$, this leads to $\mu_{k, l} \mathcal{Q}_{k-1, l} \mathbb{T}_{k-1, l}^{k, l} f=0$, which concludes the proof.

In view of Proposition 4, the following implication holds:

$$
f \in \operatorname{Ker}_{h} \mathcal{Q}_{k, l} \Rightarrow \underline{\partial}_{x} f=\mu_{k, l} g_{1}+\nu_{k, l} g_{2}
$$

with $g_{1} \in \operatorname{Ker}_{h-1} \mathcal{Q}_{k-1, l}$ and $g_{2} \in \operatorname{Ker}_{h-1} \mathcal{Q}_{k, l-1}$. Conversely, let $f \in \mathcal{C}^{\infty}\left(\mathbb{R}^{m}, \mathcal{S}_{k, l}\right)$ now be a polynomial, $h$-homogeneous in $\underline{x}$, such that $g_{1}$ and $g_{2}$ satisfy the requirements mentioned above and with

$$
\underline{\partial}_{x} f=\mu_{k, l} g_{1}+\nu_{k, l} g_{2},
$$

then also $f \in \operatorname{Ker}_{h} \mathcal{Q}_{k, l}$. Now we would like to investigate whether for any choice of these polynomials $g_{1}$ and $g_{2}$, there indeed exists a polynomial $f$ satisfying (10). In other words, we are trying to characterize the conditions which have to be imposed on $g_{1} \in \operatorname{Ker}_{h-1} \mathcal{Q}_{k-1, l}$ and $g_{2} \in \operatorname{Ker}_{h-1} \mathcal{Q}_{k, l-1}$, such that the following equivalence holds:

$$
f \in \operatorname{Ker}_{h} \mathcal{Q}_{k, l} \Leftrightarrow\left\{\begin{array}{l}
\underline{\partial}_{x} f=\mu_{k, l} g_{1}+\nu_{k, l} g_{2} \\
\underline{\partial}_{u} f=0 \\
\underline{\partial}_{v} f=0 \\
\left\langle\underline{u}_{,} \underline{\partial}_{v}\right\rangle f=0
\end{array} .\right.
$$

Just like for the Rarita-Schwinger case, see [7], this requires the study of compatibility conditions for an inhomogeneous system of equations involving three Dirac operators. The system above is not of the form considered in [9] due to the presence of the last equation. We will split this system into a simplified system and an extended system. The simplified system (denoted SiSy) is given by

$$
\text { SiSy } \leftrightarrow\left\{\begin{array}{rl}
\underline{\partial}_{x} f & =\mu_{k, l} g_{1}+\nu_{k, l} g_{2} \\
\underline{\partial}_{u} f & =0 \\
\underline{\partial}_{v} f & =0
\end{array},\right.
$$

whereas adding the extra condition $\left\langle\underline{u}, \underline{\partial}_{v}\right\rangle f=0$ defines the extended system (denoted ExSy). The next proposition tells us it is sufficient to study solutions for SiSy:

Proposition 5. Let $f \in \mathcal{P}_{h, k, l}(\mathbb{S})$ be a solution of SiSy. Then the projection $\pi(f)$ of $f$ on the kernel of $\left\langle\underline{u}, \underline{\partial}_{v}\right\rangle$ satifies ExSy:

$$
\left\{\begin{array}{l}
\underline{\partial}_{x} \pi(f)=\mu_{k, l} g_{1}+\nu_{k, l} g_{2} \\
\underline{\partial}_{u} \pi(f)=0 \\
\underline{\partial}_{v} \pi(f)=0 \\
\left\langle\underline{u}_{,}, \underline{\partial}_{v}\right\rangle \pi(f)=0
\end{array} .\right.
$$


Proof. Using the Fischer decomposition with respect to the operator $\left\langle\underline{u}, \underline{\partial}_{v}\right\rangle$, we can write any solution $f$ of SiSy as

$$
f=f_{k, l}+\left\langle\underline{v}, \underline{\partial}_{u}\right\rangle f_{k+1, l-1}+\ldots+\left\langle\underline{v}, \underline{\partial}_{u}\right\rangle^{l} f_{k+l, 0}=\sum_{j=0}^{l}\left\langle\underline{v}, \underline{\partial}_{u}\right\rangle^{j} f_{k+j, l-j}
$$

with $f_{k+j, l-j}$ in $\operatorname{Ker}\left\langle\underline{u}, \underline{\partial}_{v}\right\rangle \subset \mathcal{P}_{h, k, l}(\mathbb{S})$, for all $j=0, \ldots, l$. Define the projection map $\pi$ by

$$
\pi: \mathcal{P}_{h, k, l}(\mathbb{S}) \rightarrow \operatorname{Ker}\left\langle\underline{u}, \underline{\partial}_{v}\right\rangle: f \mapsto \pi(f)=f_{k, l} .
$$

We will prove that $f_{k, l}$ satisfies ExSy. Because $\underline{\partial}_{u} f=0$ and $\left[\underline{\partial}_{u},\left\langle\underline{v}, \underline{\partial}_{u}\right\rangle\right]=0$, we already have that $\underline{\partial}_{u} f_{k+j, l-j}=0$. Combining this result with $\underline{\partial}_{v} f=0$, which means that also the commutator $\left[\underline{\partial}_{v},\left\langle\underline{v}, \underline{\partial}_{u}\right\rangle\right]=\underline{\partial}_{u}$ acts trivially, we find that $\underline{\partial}_{v} f_{k+j, l-j}=0$ holds too. Finally, we verify that $\underline{\partial}_{x} f_{k, l}=\mu_{k, l} g_{1}+\nu_{k, l} g_{2}$. As $\left[\underline{\partial}_{x},\left\langle\underline{u}, \underline{\partial}_{v}\right\rangle\right]=0$, it is easily seen that $\pi\left(\underline{\partial}_{x} f\right)=\underline{\partial}_{x} \pi(f)$ and hence

$$
\underline{\partial}_{x} f_{k, l}=\underline{\partial}_{x} \pi(f)=\pi\left(\underline{\partial}_{x} f\right)=\pi\left(\mu_{k, l} g_{1}+\nu_{k, l} g_{2}\right)=\mu_{k, l} g_{1}+\nu_{k, l} g_{2}
$$

because $\mu_{k, l} g_{1}+\nu_{k, l} g_{2} \in \operatorname{Ker}\left\langle\underline{u}, \underline{\partial}_{v}\right\rangle$ by construction. Note that $f_{k, l} \neq 0$, since otherwise $\mu_{k, l} g_{1}+\nu_{k, l} g_{2}=0$.

To any inhomogeneous system of Dirac equations of the form

$$
\left\{\begin{array}{l}
\underline{\partial}_{x} f=h_{1} \\
\underline{\partial}_{u} f=h_{2} \\
\underline{\partial}_{v} f=h_{3}
\end{array}\right.
$$

corresponds the following set of compatibility conditions, see [9]:

$$
\left\{\begin{array}{l}
\Delta_{u} h_{1}+\underline{\partial}_{x} \underline{\partial}_{u} h_{2}=0 \\
\Delta_{v} h_{1}+\underline{\partial}_{x} \underline{\partial}_{v} h_{3}=0 \\
\Delta_{v} h_{2}+\underline{\partial}_{u} \underline{\partial}_{v} h_{3}=0 \\
\Delta_{x} h_{2}+\underline{\partial}_{u} \underline{\partial}_{x} h_{1}=0 \\
\Delta_{x} h_{3}+\underline{\partial}_{v} \underline{\partial}_{x} h_{1}=0 \\
\Delta_{u} h_{3}+\underline{\partial}_{v} \underline{\partial}_{u} h_{2}=0 \\
\left\{\underline{\partial}_{x}, \underline{\partial}_{u}\right\} h_{3}=\underline{\partial}_{v}\left(\underline{\partial}_{x} h_{2}+\underline{\partial}_{u} h_{1}\right) \\
\left\{\underline{\partial}_{u}, \underline{\partial}_{v}\right\} h_{1}=\underline{\partial}_{x}\left(\underline{\partial}_{u} h_{3}+\underline{\partial}_{v} h_{2}\right) \\
\left\{\underline{\partial}_{v}, \underline{\partial}_{x}\right\} h_{2}=\underline{\partial}_{u}\left(\underline{\partial}_{v} h_{1}+\underline{\partial}_{x} h_{3}\right)
\end{array}\right.
$$

The last three relations (which are linear dependent) are the radial algebra relations, which were investigated in [19]. In our present case of interest, we have to put $h_{1}=\mu_{k, l} g_{1}+\nu_{k, l} g_{2}$ and $h_{2}=0=h_{3}$. Motivated by the Rarita-Schwinger case, we will split these conditions into two sets. First of all, define the compatibility conditions of type I (denoted CC-I):

$$
\left\{\begin{array}{l}
\Delta_{u}\left(\mu_{k, l} g_{1}+\nu_{k, l} g_{2}\right)=0 \\
\Delta_{v}\left(\mu_{k, l} g_{1}+\nu_{k, l} g_{2}\right)=0 \\
\underline{\partial}_{u} \underline{\partial}_{v}\left(\mu_{k, l} g_{1}+\nu_{k, l} g_{2}\right)=0 \\
\underline{\partial}_{v} \underline{\partial}_{u}\left(\mu_{k, l} g_{1}+\nu_{k, l} g_{2}\right)=0 \\
\left\{\underline{\partial}_{u}, \underline{\partial}_{v}\right\}\left(\mu_{k, l} g_{1}+\nu_{k, l} g_{2}\right)=0
\end{array}\right.
$$


together with the extra condition (which is the one leading to the ExSy)

$$
\left\langle\underline{u}, \underline{\partial}_{v}\right\rangle\left(\mu_{k, l} g_{1}+\nu_{k, l} g_{2}\right)=0 .
$$

We are then left with two compatibility conditions of type II (denoted CC-II):

$$
\left\{\begin{array}{ll}
\underline{\partial}_{u} \underline{\partial}_{x}\left(\mu_{k, l} g_{1}+\nu_{k, l} g_{2}\right)=0 & \text { (i) } \\
\underline{\partial}_{v} \underline{\partial}_{x}\left(\mu_{k, l} g_{1}+\nu_{k, l} g_{2}\right)=0 & \text { (ii) }
\end{array} .\right.
$$

Note that they are not independent, because

$$
\begin{aligned}
\underline{\partial}_{u} \underline{\partial}_{x}\left(\mu_{k, l} g_{1}+\nu_{k, l} g_{2}\right)=0 & \Rightarrow\left\langle\underline{u}, \underline{\partial}_{v}\right\rangle \underline{\partial}_{u} \underline{\partial}_{x}\left(\mu_{k, l} g_{1}+\nu_{k, l} g_{2}\right)=0 \\
& \Leftrightarrow \underline{\partial}_{v} \underline{\partial}_{x}\left(\mu_{k, l} g_{1}+\nu_{k, l} g_{2}\right)=0 .
\end{aligned}
$$

This means that it is sufficient to check CC-II (i). However, it will turn out to be useful to investigate both conditions anyway.

We mentioned before that in the case of the Rarita-Schwinger operator, the analysis of compatibility conditions leads to the conclusion that the kernel space for $\mathcal{R}_{k-1}$ can be embedded into the kernel space for $\mathcal{R}_{k}$. The compatibility conditions exactly determine the structure of the kernel space (i.e. the type B solutions).

In the present case of the operator $\mathcal{Q}_{k, l}$, it is not difficult to show that the conditions of CC-I are equivalent with $g_{1}$ and $g_{2}$ being elements of $\mathcal{C}^{\infty}\left(\mathbb{R}^{m}, \mathcal{S}_{k-1, l}\right)$ and $\mathcal{C}^{\infty}\left(\mathbb{R}^{m}, \mathcal{S}_{k, l-1}\right)$ respectively. In other words, these conditions again fix the values. However, the conditions of CC-II are not equivalent with every $g_{1} \in \operatorname{Ker}_{h-1} \mathcal{Q}_{k-1, l}$ and every $g_{2} \in \operatorname{Ker}_{h-1} \mathcal{Q}_{k, l-1}$. Indeed, we will prove that only for $g_{1}$ and $g_{2}$ satisfying

$$
\mathbb{T}_{k-1, l-1}^{k-1, l} g_{1}=\frac{(m+2 l-4)(k-l+2)}{(m+2 k-2)(k-l+1)} \mathbb{T}_{k-1, l-1}^{k, l-1} g_{2}
$$

there exists a polynomial $f$ in $\operatorname{Ker}_{h} \mathcal{Q}_{k, l}$ such that $\underline{\partial}_{x} f=\mu_{k, l} g_{1}+\nu_{k, l} g_{2}$. In particular, this relation is satisfied for $g_{1}$ and $g_{2}$ in the kernel of $\mathbb{T}_{k-1, l-1}^{k-1, l}$ and $\mathbb{T}_{k-1, l-1}^{k, l-1}$ respectively. Note that by Proposition 4, both the left- and right-hand side of (11) are polynomials in $\operatorname{Ker}_{h-2} \mathcal{Q}_{k-1, l-1}$.

Demanding that CC-II (ii) is satisfied, leads to the stated relation between $g_{1}$ and $g_{2}$ :

$$
\begin{aligned}
\underline{\partial}_{v} \underline{\partial}_{x}\left(\underline{u} g_{1}\right)= & -\underline{\partial}_{v} \underline{\partial}_{x}\left(\underline{v}-\frac{\underline{u}}{\left.k-l+\underline{v}_{u}\right\rangle}\right) g_{2} \\
\Leftrightarrow-2 \underline{u}\left\langle\underline{\partial}_{v}, \underline{\partial}_{x}\right\rangle g_{1}= & 2 \underline{v}\left\langle\underline{\partial}_{v}, \underline{\partial}_{x}\right\rangle g_{2}-(m+2 l-6) \underline{\partial}_{x} g_{2} \\
& \left.-\frac{2}{k-l+1} \underline{u}_{\underline{\underline{\partial}}}, \underline{\partial}_{x}\right\rangle g_{2}-2 \frac{\underline{u}\left\langle\underline{v}, \underline{\partial}_{u}\right\rangle\left\langle\underline{\partial}_{v}, \underline{\partial}_{x}\right\rangle}{k-l+1} g_{2} \\
\Leftrightarrow-2 \underline{u}\left\langle\underline{\partial}_{v}, \underline{\partial}_{x}\right\rangle g_{1}= & \left.-2 \frac{(m+2 l-4)(k-l+2)}{(m+2 k-2)(k-l+1)} \underline{u}\left[\underline{\partial}_{u}, \underline{\partial}_{x}\right\rangle+\frac{\underline{u}\left\langle\underline{v}_{\underline{\partial}} \underline{\partial}_{u}\right\rangle\left\langle\underline{\partial}_{v}, \underline{\partial}_{x}\right\rangle}{k-l+2}\right] g_{2} \\
\Leftrightarrow \quad \mathbb{T}_{k-1, l-1}^{k-1, l} g_{1}= & \frac{(m+2 l-4)(k-l+2)}{(m+2 k-2)(k-l+1)} \mathbb{T}_{k-1, l-1}^{k, l-1} g_{2},
\end{aligned}
$$


where we have used explicitly that $\mathcal{Q}_{k, l-1} g_{2}=0$. For $g_{1}$ and $g_{2}$ satisfying this relation, further calculations then show that CC-II (i) holds as well, i.e.

$$
\underline{\partial}_{u} \underline{\partial}_{x}\left[\underline{u} g_{1}+\left(\underline{v}-\frac{\underline{u}\left\langle\underline{v}, \underline{\partial}_{u}\right\rangle}{k-l+1}\right) g_{2}\right]=0 \text {. }
$$

Summarizing, the type B solutions of the operator $\mathcal{Q}_{k, l}$ can be of the following type:

(i) choosing $g_{2}=0$, we have that $\operatorname{Ker}_{h-1} \mathcal{Q}_{k-1, l} \cap \operatorname{Ker}_{k-1, l-1}^{k-1, l} \subset \operatorname{Ker}_{h} \mathcal{Q}_{k, l}$;

(ii) choosing $g_{1}=0$, we have that $\operatorname{Ker}_{h-1} \mathcal{Q}_{k, l-1} \cap \operatorname{Ker}_{k-1, l-1}^{k, l-1} \subset \operatorname{Ker}_{h} \mathcal{Q}_{k, l}$;

(iii) finally, choosing both $g_{1}$ and $g_{2} \neq 0$ is only possible if relation (11) is satisfied, which amounts to saying that certain elements in $\operatorname{Ker}_{h-2} \mathcal{Q}_{k-1, l-1}$ can also be inverted. This behaviour is different from what was obtained for the classical Rarita-Schwinger case, and is of course expected to hold in the more general case too. These summands could be described as $\operatorname{Im} \mathbb{T}_{k-1, l-1}^{k, l-1} \cap \operatorname{Im} \mathbb{T}_{k-1, l-1}^{k-1, l} \subset \operatorname{Ker}_{h-2} \mathcal{Q}_{k-1, l-1}$.

In the special case $k=l$, there exists only one twistor operator and (11) reduces to

$$
\mathbb{T}_{k-1, k-1}^{k, k-1} g_{2}=0 .
$$

The type B solutions of the operator $\mathcal{Q}_{k, k}$ are thus equivalent with elements of

$$
\operatorname{Ker}_{h-1} \mathcal{Q}_{k, l-1} \cap \operatorname{Ker} \mathbb{T}_{k-1, k-1}^{k, k-1} .
$$

In both cases, we have thus obtained an inductive procedure to describe (at least formally) the space of polynomial solutions for the operator $\mathcal{Q}_{k, l}$. In [2], we have proved this using a dimensional analysis, while in [3] we have constructed the explicit embedding factors realizing the decomposition of the kernel.

\section{References}

[1] Brackx, F., Delanghe, R., Sommen, F., Clifford Analysis, Research Notes in Mathematics 76, Pitman, London (1982).

[2] Brackx, F., Eelbode, D., Van de Voorde, L., The polynomial null solutions of a higher spin Dirac operator in two vector variables, submitted.

[3] Brackx, F., Eelbode, D., Van de Voorde, L., The fundamental solution of a higher spin Dirac operator in two vector variables, submitted.

[4] Brackx, F., Eelbode, D., Raeymaekers, T., Van de Voorde, L., Triple monogenic functions and higher spin Dirac operators, submitted.

[5] Branson, T., Stein-Weiss operators and ellipticity, J. Funct. Anal. 151 No. 2 (1997), pp. 334-383. 
[6] Bureš, J., The higher spin Dirac operators, in Differential geometry and applications, Masaryk Univ. Brno (1999), pp. 319-334.

[7] Bureš, J., Sommen, F., Souček, V., Van Lancker, P., Rarita-Schwinger type operators in Clifford analysis, Journal of Funct. Anal. 185 (2001), pp. 425-456.

[8] Bureš, J., Sommen, F., Souček, V., Van Lancker, P., Symmetric analogues of RaritaSchwinger equations, Ann. Glob. Anal. Geom. 21 No. 3 (2001), pp. 215-240.

[9] Colombo, F., Sabadini, I., Sommen, F., Struppa, D. C., Analysis of Dirac Systems and Computational Algebra, Progress in Mathematical Physics, Vol. 39, Birkhäuser (2004).

[10] Constales, D., Sommen. F., Van Lancker, P., Models for irreducible representations of Spin $(m)$, Adv. Appl. Clifford Algebras 11 No. S1 (2001), pp. 271-289.

[11] Delanghe, R., Sommen, F., Souček, V., Clifford analysis and spinor valued functions, Kluwer Academic Publishers, Dordrecht (1992).

[12] Eelbode, D., Van de Voorde, L., Higher spin operators and polyharmonic functions, Proceedings of the 16th ICFIDCAA, Korea (2008), pp. 137-142.

[13] Fegan, H., D., Conformally invariant first order differential operators, Quart. J. Math. 27 (1976), pp. 513-538.

[14] Fulton, W., Harris, J., Representation theory : a first course, Springer-Verlag, New York (1991).

[15] Gilbert, J., Murray, M.A.M., Clifford algebras and Dirac operators in harmonic analysis, Cambridge University Press, Cambridge (1991).

[16] Humphreys, J., Introduction to Lie algebra and representation theory, SpringerVerlag, New York (1972).

[17] Lawson, H.B., Michelsohn, M-L., Spin Geometry, Princeton University Press, Princeton, 1989.

[18] Molev, A.I., Yangians and classical Lie algebras, Mathematical Surveys and Monographs 143. American Mathematical Society, Providence (2007).

[19] Sabadini, I., Sommen, F., Struppa, D., The Dirac complex on abstract vector variables: megaforms, Exp. Math. Vol. 12 No. 3 (2003), pp. 351-364.

[20] Severa, V., Invariant differential operators between spinor-valued forms, $\mathrm{PhD}$-thesis, Charles University, Prague.

[21] Slovak, J., Natural operators on conformal manifolds, Masaryk University Dissertation, Brno (1993).

[22] Stein, E.W., Weiss, G., Generalization of the Cauchy-Riemann equations and representations of the rotation group, Amer. J. Math. 90 (1968), pp. 163-196. 